\title{
Indutores de alinhamento estratégico: comparações preliminares sobre valor entre empresas de capital privado e empresas estatais no Brasil
}

\author{
José Francisco de Carvalho Rezende \\ Programa de Pós-Graduação em Administração/Universidade do Grande Rio (UNIGRANRIO) \\ Joaquim Rubens Fontes Filho \\ Escola Brasileira de Administração Pública e de Empresas/Fundação Getulio Vargas (EBAPE/ \\ FGV)
}

\begin{abstract}
O artigo trata da comparação da eficiência entre empresas privadas e estatais, discutindo diferenças dos procedimentos de alinhamento estratégico e criação de valor utilizados, e apresentando evidências sobre os "tipos" de alinhamento estratégico e os "modos" de criação de valor utilizados. O estudo utiliza informações de survey aplicado anteriormente à amostra de 95 organizações brasileiras, estratificadas segundo a origem do capital em privadas ou estatais. Foram testadas hipóteses sobre diferenças de médias nos vetores de alinhamento estratégico, percentuais distintos no tocante a padrões de originação de valor e comportamento das componentes do valor total. Os testes apresentaram, de forma recorrente, diferenças de médias em favor das empresas privadas, segundo intervalo de confiança superior a 85\%, nível considerado adequado a um estudo de natureza exploratória. No tocante à originação de valor, foi constatada performance superior das empresas de capital privado em todas as dimensões/ perspectivas. Considerando a relevância econômica e social das empresas estatais no país, os resultados do estudo podem contribuir para o desenvolvimento de novas perspectivas de análise do desempenho das organizações gerenciadas pelo Estado, levando a mecanismos de acompanhamento mais alinhados aos desafios de gestão dessas organizações.
\end{abstract}

Palavras-chave: desempenho; valor; empresas estatais brasileiras; alinhamento estratégico.

Inductores de aliñamiento estratégico: comparaciones entre empresas del estado y empresas privadas en Brasil

Este artículo presenta la comparación de la eficiencia entre las empresas privadas y las denominadas estatales. Analiza las diferencias de los procedimientos de aliñamiento estratégico y la creación de valor. Además se presenta evidencias sobre los "tipos" de aliñamiento estratégico así como los "modos"

Artigo recebido em 24 jan. 2012 e aceito em 18 fev. 2013. 
de creación de valor que son utilizados. El estudio que se presenta utiliza informaciones de survey que fueron aplicados a una muestra de 95 organizaciones brasileñas, las mismas que fueron estratificadas según el origen del capital constitutivo ya sea para empresas privadas o estatales. Fueron probados hipótesis sobre la diferencia de las medias en los vectores de aliñamiento estratégico. Porcentajes distintos en relación a los patrones que originan el valor y comportamiento de los componentes del valor total. Las pruebas presentaron de forma recurrente, diferencias de medias a favor de las empresas privadas, según el intervalo de confianza del $85 \%$, nivel que es considerado adecuado en un estudio considerado exploratorio. En relación al origen del valor, fue constatada performance superior de las empresas de capital privad en todas las dimensiones/perspectivas. Considerando la relevancia económica y social de las empresas estatales en el país los resultados del estudio pueden contribuir para el desarrollo de nuevas perspectivas de análisis de desempeño de las organizaciones gerenciadas por el Estado, sugiriendo así la utilización de mecanismos de acompañamiento mas aliñados a los desafíos de la gestión de las empresas estatales.

Palabras clave: rendimiento; valor; empresas del estado; aliñamiento estratégico.

Strategic alignment drivers: preliminary comparisons on value between private corporations and state-owned enterprises in Brazil

This paper is placed in the context of comparative analyzes of efficiency between private and state companies and discusses differences regarding the strategic alignment and value creation management procedures on those enterprises, allowing to obtain evidence about "types" of alignment and value origination management "modes". From hypothetical-deductive approach, we used information from the survey previously applied to 95 Brazilian organizations, now stratified according to the origin of capital, private or state. We tested hypotheses about the existence of mean differences in strategic alignment vectors, on the existence of different proportions of types of state and private companies with respect to patterns of value origination and the behavior of the components of the total value created. The tests presented on a recurring basis, mean differences in favor of private enterprise, with results displayed from the confidence interval above $85 \%$, satisfactory when taking into consideration that this is an exploratory study. Also regarding the origination value, was found superior performance of private equity firms in all dimensions/perspectives. Faced with the findings and the relevance of state enterprises, it is expected that the evidence to enable a new performance approach of organizations managed by the state, triggering mechanisms for monitoring performance more in line with the management challenges of contemporary organizations.

KEYWORDs: performance; value; Brazilian state owned enterprises; strategic alignment.

\section{Introdução}

Diante da complexidade crescente das organizações, principalmente quanto à necessidade e à capacidade de prestação de contas aos stakeholders, é notável o descompasso entre os sistemas tradicionais de governança orientados para aspectos tangíveis da gestão e a sofisticação das demandas no tocante à originação e ao compartilhamento de valor entre as partes interessadas. Especificamente para organizações que têm origem e operam com aporte de capital público, as expectativas seriam ainda maiores em vista do imperativo de alocação 
dos recursos do Estado em atividades que possibilitassem a implementação otimizada de políticas públicas.

No caso brasileiro, há longa tradição de se atribuir às empresas estatais um nível de eficiência menor do que o quantum obtido por uma congênere privada. Se, por um lado, estudos de Boardman e Vining (1989) e de Dewenter e Malatesta (2001) apontam a prevalência da capacidade de gestão privada sobre o estado empresário, autores como Bozec, Breton e Côté (2002) pontuam a possível importância da presença estatal para atenuar problemas de desemprego, apoiar manifestações populares, controle de preços ou fortalecimento de setores da economia nacional.

Este estudo busca contribuir para o fortalecimento da discussão sobre desempenho das empresas estatais brasileiras, a partir de proposições que retratam componentes de sua estrutura e dinâmica organizacional (Rezende e Nogueira, 2010), no momento de escolha de novas alternativas sobre a presença do estado na gestão de ativos de produção que sucede a ênfase em privatizações dos anos 1980 e 1990. Nesse sentido, no campo de estudos relacionados à governança e performance estratégica das organizações, esta pesquisa busca identificar a existência de padrões diferenciados de gestão dos indutores (proposições, fatores e vetores) de alinhamento estratégico, direta ou indiretamente associados a diferenças na originação de valor em empresas de capital privado e estatais.

As análises permitiram identificar que a falta de aderência na aplicação de vetores de alinhamento estratégico pode afetar tanto empresas de capital privado quanto estatais, sendo estas mais sujeitas a descontinuidades na intensidade, direção e ponto de aplicação de tais vetores por conta da alternância política e de ajustes de curso das diretrizes de governo.

\section{Panorama institucional das empresas estatais no Brasil}

A denominação de empresa estatal federal, conforme disposto no Decreto-Lei no 200/1967, é atribuída às entidades da administração indireta, dotadas de personalidade jurídica própria, de direito privado, com patrimônio próprio e com capital exclusivo ou de maioria da União, criada por lei para desempenhar atividades de natureza empresarial que o governo deve exercer. Subdividem-se em empresas públicas, quando o capital é exclusivo da União, ou sociedade de economia mista, quando a maioria das ações com direito a voto pertence diretamente à União ou a entidades da administração indireta.

Ainda conforme esse decreto-lei, sua vinculação se dá ao ministério que abrange a área de competência de sua principal atividade. Entretanto, a estrutura de governança das estatais distribui as competências na supervisão e controle dessas empresas em outras duas áreas, o Ministério do Planejamento, Orçamento e Gestão (MPOG) e o Ministério da Fazenda (MF). O MPOG, por meio do Departamento de Coordenação e Governança das Empresas Estatais

(Dest), é responsável pela elaboração do Programa de Dispêndios Globais e da proposta do Orçamento de Investimentos das estatais, assim como pelo acompanhamento e disponibilização de informações econômico-financeiras e manifestação sobre propostas dessas empresas 
quanto a alterações na sua política salarial, nos planos de previdência e no quantitativo de empregados. Ao MF, por meio da Secretaria do Tesouro Nacional, cabe a administração dos valores mobiliários representativos de participações da União, o que envolve os interesses societários em empresas estatais.

O Conselho de Administração das empresas estatais deve contar, além da representação de acionistas minoritários, quando for o caso, com membro indicado pelo Ministério do Planejamento (MP) e os demais indicados pelo ministério supervisor. Mais recentemente, foi introduzida a obrigatoriedade da participação de representantes dos trabalhadores nos conselhos de administração das empresas estatais, inclusive controladas, com mais de 200 empregados. No Conselho Fiscal, além dos indicados pelo ministério supervisor, cabe ao Tesouro Nacional indicar um representante e acompanhar, orientar e avaliar sua atuação, direito extensível às empresas em que a União tenha participação minoritária no capital.

O número de empresas estatais federais, que na década de 1980 era de 215, chegou a 258 na década seguinte e, após sucessivas privatizações, alcançava 115 em dezembro de 2006, registrando então um total de 398.852 funcionários. Ao final de 2009 havia um total de 481,8 mil empregados registrados no quadro de pessoal próprio no conjunto das estatais federais, um aumento de aproximadamente $21 \%$ em três anos. No ano de 2009, os dispêndios totais das empresas estatais federais atingiram o volume de R 696 bilhões (Brasil, 2010b).

Segundo a OECD (2004b), a operação das empresas estatais diferencia-se das privadas por estarem sujeitas a menos restrições orçamentárias, por operarem frequentemente sob menor pressão competitiva, por distintos padrões de transparência e prestação de contas, e por uma evidenciação muitas vezes difusa da figura do proprietário ou controlador entre os vários ministérios.

Além disso, governança corporativa das estatais apresenta fragilidades, principalmente em razão da pouca clareza dos objetivos empresariais, resultante da influência política em suas definições estratégicas e operações, o que dificulta uma análise objetiva de sua gestão e resultados empresariais.

Na perspectiva do acompanhamento do desempenho, podem ser consideradas questões basilares para compreender a atuação das empresas estatais em face das empresas privadas: (i) a transparência nos dados para avaliação dos resultados; (ii) a capacidade de separar os custos do negócio daqueles impostos pela execução de políticas governamentais; (iii) e a eficiência comparada.

Conforme matéria do jornal O Globo (Doca, 2010), a Controladoria Geral da União identificou que 10 empresas estatais, em um universo de 60, não cumpriam normas do próprio governo ou disponibilizavam página específica no endereço eletrônico para dar publicidade aos gastos, estando essa obrigação já disciplinada em lei.

A dificuldade na transparência da situação do negócio, acrescida então pela mescla dos custos do negócio aos custos políticos não relacionados, contribui para o questionamento da eficiência das empresas estatais diante das congêneres do setor privado, objeto de extensa literatura, embora pouco conclusiva. Conforme Bozec, Breton e Côté (2002), embora a maioria dos estudos comparativos sugira maior eficiência da empresa privada, apresentam deficiências 
no delineamento do objeto da pesquisa ao não reconhecerem a diferença de objetivos da empresa estatal. Em análise do período 1976-96, notaram evidências que, tendo como referência o objetivo de otimização dos resultados, a empresa estatal tem desempenho semelhante ao da privada. Analisam que o desempenho inferior da estatal encontrado por outros autores pode resultar de falhas metodológicas.

Ao contrário, autores como Boardman e Vining (1989) e Dewenter e Malatesta (2001) apontam o melhor desempenho da empresa privada. Boardman e Vining (1989) utilizam a teoria do direito de propriedade da firma para analisar que a empresa estatal tende a ter desempenho inferior, o que concluem empiricamente observando amostra de 500 empresas não financeiras de fora dos Estados Unidos. Na mesma linha, Dewenter e Malatesta (2001) identificaram evidências fortes de um desempenho inferior da empresa estatal. Avaliam que, ao reduzirem a importância de objetivos de desempenho e lucratividade em prol de objetivos políticos ou sociais, e pela pouco frequente distribuição de lucros, o ambiente das estatais tende a reduzir a eficiência do monitoramento sobre os gestores e produzir uma degradação do desempenho. Contudo, ao avançarem os estudos para verificar o efeito da privatização sobre a lucratividade das empresas, observaram que o aumento de produtividade foi amplamente evidenciado durante os três anos anteriores à privatização e diminuído nos cinco anos subsequentes. Para os autores, esses dados sugerem que os governos são capazes de configurar empresarialmente suas empresas e orientá-las para produzir um desempenho adequado.

Para Andrés, Guasch e Azumendi (2011), após as privatizações dos anos 1980 e 1990, pesquisadores voltaram a discutir a empresa estatal em função de aspectos da governança corporativa mais do que em função do papel do estado. Esses autores identificaram, em amostras de empresas estatais latino-americanas dos setores de água e energia, a predominância de tipos de organizações ainda afastadas dos conceitos basilares da New Public Management, com grande ênfase para a falta de adequação de instrumentos de formulação, desdobramento, acompanhamento, responsabilização e remuneração dos gestores e dos profissionais com base na estratégia definida.

Embora as empresas estatais sejam aparentemente resguardadas de problemas como falência ou tomada hostil de controle, a complexidade da rede de stakeholders, com interesses nem sempre identificados, leva à multiplicidade de expectativas, responsabilidades e metas, sendo este um dos grandes problemas na gestão de empresas estatais: além de enfrentar os problemas comuns a todas as corporações, há o problema da diluição da figura do principal e há, por parte dos gestores, um desalinhamento sobre a interpretação de "interesse público", questão maximizada em governos de coalizão (Miranda e Amaral, 2011).

Aspectos complementares sobre o desempenho das empresas estatais são discutidos por Hertog (2010), ao estudar seus resultados em países dependentes de recursos extrativistas. A partir do modelo apresentado na figura 1 (Hertog, 2010:280), propõe uma nova agenda em que não haveria, a priori, impedimentos à atuação do Estado na gestão de ativos do setor produtivo, atendidas as salvaguardas em relação ao populismo e ao controle excessivamente centralizado. 
Figura 1

Efeitos do populismo e do controle administrativo centralizado em empresas estatais

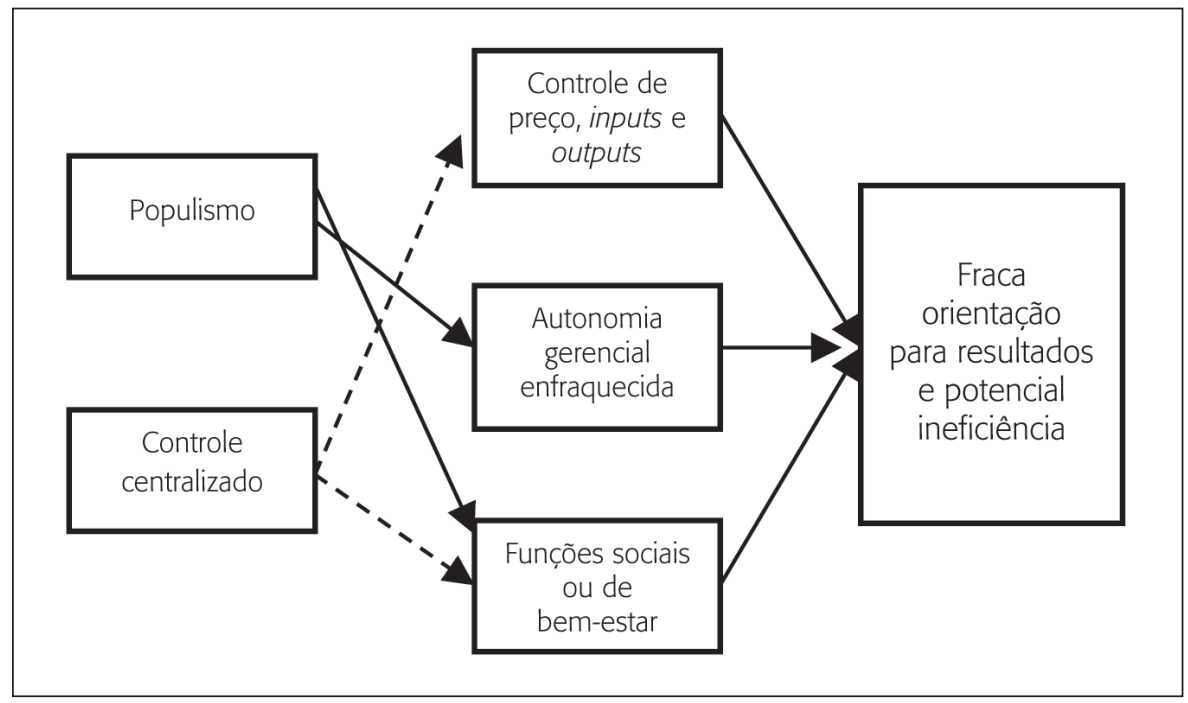

Fonte: Hertog (2010).

\section{Alinhamento estratégico}

O novo pano de fundo empresarial vem sendo caracterizado alternadamente como sociedade da informação (Shapiro e Varian, 1998), do conhecimento (Crawford, 1994) ou da economia digital (Tapscott, 1995), e a diferenciação dos termos derivaria do poder de apropriação do valor gerado por ativos intangíveis e sua objetivação, de acordo com o contexto, em conhecimento intercambiável.

Para Nonaka e Takeuchi (1997), a objetivação do conhecimento estaria vinculada a processos coletivos de socialização, externalização, combinação e internalização, modos necessários à criação de conhecimento com alto poder de inovação e potencial para a geração de valor. Sveiby (1994) avalia o mecanismo de introdução - capacidade de continuamente recombinar e ressignificar o conhecimento - como um dos principais vetores de diferenciação acessíveis às organizações. Assim, a concepção de novas sistemáticas para acompanhamento da performance com base na integração de enfoques sobre desempenho é debatida por Bukh e colaboradores (2002:30), tendo como propósito discutir a compatibilidade entre o Balanced Scorecard e os painéis de apoio à Gestão do Capital Intelectual nas organizações.

A capacidade de coordenar os esforços empresariais para garantir o equilíbrio de interesses inerente a uma opção estratégica, assim como a possibilidade de testar as hipóteses que dão origem à estratégia, vêm se mostrando como um diferencial importante para a melhor alocação de recursos. 
Além disso, a participação crescente dos intangíveis no valor dos empreendimentos da chamada economia do conhecimento (Crawford, 1994; Kaplan e Norton, 2003) parece ter alcançado níveis de fundamentação empírica (Sveiby, 1994; Bontis, Crossan e Hulland, 2002; Ballow, Burgman e Molnar, 2004) que justificam a adoção de sistemáticas de gestão que consigam melhor capturar e traduzir o valor do capital intelectual que se torna disponível pela acumulação da riqueza inerente aos processos, aos relacionamentos, à força de trabalho, à competência em inovar e à capacidade de se manter numa trajetória equilibrada em face das alterações nos níveis de turbulência.

Kaplan e Norton (2003) apontam que dois terços das organizações por eles estudadas não criam forte alinhamento entre suas estratégias e os programas de recursos humanos e de tecnologia da informação, levando à perda na eficiência dos investimentos uma vez que estes não seriam capazes de colocar a estratégia em ação. Os ativos intangíveis passariam a exercer influência tal para o sucesso da estratégia formulada que seriam necessárias três abordagens básicas para melhorar o alinhamento estratégico como um todo: (i) desenvolvimento dos cargos com real poder de contribuição para a estratégia; (ii) criação de um portfólio estratégico de tecnologia da informação; e (iii) estabelecimento de uma agenda de mudança organizacional.

A complexidade crescente - tanto do ambiente, quanto das soluções estratégicas e estruturais adotadas pelas organizações - aparentemente vem demandando maior capacidade de lidar com abordagens sistêmicas e capazes de fornecer amplo feedback sobre valor. O entendimento da organização como entidade viva (De Geus, 1998) repercutiria na identificação de sua capacidade criadora como principal elemento de diferenciação.

Para Shapiro e Varian (1998) e Hax e Wilde II (1999), as novas abordagens deveriam passar pela exploração do potencial de um mercado em rede, do gerenciamento permanente das parcerias, da possibilidade de estabelecimento de travamentos ao longo das etapas de criação de valor — lock in e lock out - pela conquista antecipada de uma posição dominante em relação a um padrão adotado e pela capacidade de conquistar reputação tanto entre os empreendimentos já estabelecidos quanto junto aos emergentes.

Grouard e Meston (2001) argumentam que uma nova complexidade do ambiente vem sendo o motor da mudança organizacional em nível estratégico. A busca da estabilidade teria sido substituída pela busca da fluidez, com reflexos nos modelos de gerenciamento do desempenho empresarial e individual. A combinação entre forças internas e externas estabeleceria uma nova dinâmica de mudança com base na velocidade, na profundidade e no enfoque - questão que remeteria, de certo modo, ao conceito de vetor estratégico defendido por Norton $(1987,1995)$ no enfoque Benefits Based Planning para a definição de investimentos em tecnologia da informação. Ainda segundo Grouard e Meston (2001), a complexidade organizacional adviria das incontáveis combinações entre ativos tangíveis (terrenos, edificações, maquinário, equipamentos), ativos negociáveis (patentes, marcas, know-how) e recursos humanos, e o gerenciamento de tal diversidade passaria, necessaria- 
mente, pela obtenção de um equilíbrio satisfatório entre consistência, ambiguidade, uniformidade e disparidade.

Ethiraj e Levinthal (2008) demonstraram, por meio de experimentos computacionais, que a imposição de medidas de desempenho pouco correlacionadas leva ao desempenho abaixo do possível em vista de os atores não serem capazes de identificar cursos de ações alternativos que otimizem o conjunto de objetivos. Argumentam que em um mundo de agentes com racionalidade limitada, orientações mais simples para a ação, mesmo que forneçam apenas um subconjunto de metas, são mais eficazes para dirigir e coordenar do que representações excessivamente detalhadas de objetivos. Competiria à gestão, no que toca ao alinhamento de objetivos, proporcionar um grau de clareza e de focalização num mundo complexo.

\section{Originação de valor}

O enfoque de De Geus (1998), assim como o de Allee (2000), vêm ganhando adeptos e demarcando um tempo para o management em que o potencial de geração e de sustentação de valor estaria fundamentado na importância que é dada ao fator humano, aos intangíveis e aos relacionamentos desenvolvidos ao longo e em torno da organização.

Roos e colaboradores (1997:vii) demarcam a importância da originação, da mensuração e da gestão do capital intelectual como nova linguagem e conjunto de técnicas para a identificação das capacidades que levarão às futuras receitas e geração de valor pelas organizações, apontando para o fato de que se trata de uma nova mentalidade gerencial. Os autores propõem a existência de raízes para o capital intelectual (CI) com base em duas grandes vertentes - estratégia e mensuração — lembrando que, já em 1969, J. K. Galbraith cunhara, em carta ao economista Michael Kalecki, o conceito de CI atrelado à "ação intelectual” capaz de gerar valor para as organizações (Serenko e Bontis, 2004).

Para Kaplan e Norton (2001:11), a capacidade de implementar a estratégia seria mais importante do que a qualidade da estratégia em si. Os autores baseiam tal proposição no estudo desenvolvido pela Ernst Young junto a 275 executivos - Measures that matter, Boston, 1998 - , que constata a falha de 90\% das estratégias; 30\% devido à qualidade intrínseca e 70\% a falhas de implementação. Assim, a criação e a implementação de estratégias que geram valor a partir de intangíveis - principalmente o conhecimento - dependeriam da adoção de ferramentas de gestão que permitam executar a estratégia formulada, mais sutil e difusa do que a estratégia típica da economia industrial.

Resultados superiores foram obtidos quando as empresas passaram a ter, de fato, um foco estratégico e a alinhar os recursos para a implementação da estratégia adotada (Kaplan e Norton, 2001). Fleck (2005) desenvolve moldura teórica orientada por processos enquadrando o crescimento das organizações complexas a partir de modos de resposta aos desafios do negócio e da gestão propriamente dita. As organizações desenvolveriam capabilities 
ou liabilities que determinariam o crescimento e o sucesso no longo prazo, o que permitiria antecipar condições de autoperpetuação ou de autodestruição, argumentando que a autoperpetuação estaria condicionada pelo crescimento contínuo — viabilidade/solidez financeira, reforço positivo entre crescimento e desequilíbrio e interatividade com os stakeholders —, pela integridade organizacional - gestão da diversidade, da complexidade e dos recursos humanos - e pela existência de recursos não alocados (slack) — manobráveis para fazer frente a oportunidades e adversidades.

Roos e colaboradores (1997) apontam indicadores para a mensuração do valor de uma organização tendo por base a moldura teórica do CI e as possibilidades trazidas por uma nova e mais completa cesta de indicadores associada ao fato de que, numa situação onde o conhecimento passa a ser a tônica dos processos produtivos, a ocorrência de rendimentos crescentes põe por terra um grande número de postulados econômicos vigentes.

Defendem a criação de índices para permitir análises entre empresas, pois possibilitariam dar visibilidade a questões hoje analisadas principalmente sob o prisma do "sentimento gerencial", tais como o tempo de maturação de investimentos — defasagem entre aquisição de uma aplicação de TI e treinamento de empregados - e tradeoffs sobre um determinado investimento - maior eficiência versus satisfação dos clientes. Tais índices ampliariam a informação disponível para acionistas e investidores, trazendo maior abrangência e agregando informações complementares às demonstrações financeiras.

$\mathrm{Na}$ implementação da estratégia, índices capazes de retratar o modelo de negócio e orientar as táticas necessárias para otimizar a geração de valor (Casadesus-Masanell e Ricart, 2010) seriam fundamentais para trazer aos diversos níveis da organização uma noção mais precisa sobre sua contribuição à criação de valor.

\section{Metodologia}

Muito embora o desempenho de empresas, em geral, e das estatais, mais especificamente, seja objeto de inúmeros estudos, atribuímos à presente pesquisa o caráter exploratório (Vergara, 1998) em vista da utilização de demarcações teóricas recentes sobre vetores estratégicos e originação de valor com base nos componentes do CI (Crawford, 1994; Stewart, 1998; Sveiby, 1998; Edvinsson e Malone, 1998; Konrad Group, 2000; Bontis, Crossan e Hulland, 2002; Ballow, Burgman e Molnar, 2004; Serenko e Bontis, 2004).

A partir do problema de pesquisa proposto, se "existiriam padrões diferenciados de gestão dos indutores de alinhamento estratégico que estejam direta ou indiretamente associados a diferenças na originação de valor nas empresas privadas e nas empresas estatais", foram traçadas as três hipóteses:

、 $\mathrm{H}_{0}$, 1 : Não existem diferenças de médias nos indutores de alinhamento estratégico, desdobrados nos construtos (i) direcionamento institucional, (ii) maturidade do planejamento e 
controle; (iii) direcionamento para o futuro; (iv) direcionamento de recursos humanos; (v) direcionamento de tecnologia da informação; (vi) maturidade do gerenciamento da performance; e (vii) performance comparada;

- $\mathrm{H}_{0}$ 2: Não existem diferenças de proporções para tipos de empresas em função do padrão de alinhamento estratégico;

、 $\mathrm{H}_{0}$, 3: Não existem diferenças de média entre índices representativos do modo de originação do valor total, desdobrada em: (i) capital de relacionamentos; (ii) capital organizacional; (iii) capital humano; (iv) capital intelectual; (v) capital financeiro; e (vi) valor total.

Tomou-se, como ponto de partida, estudo que enfoca o alinhamento estratégico em empresas complexas e de repercussão na economia brasileira (Rezende e Nogueira, 2010) para, com base na estratificação a priori das empresas segundo a natureza da propriedade, discutirse a existência de diferenças de padrões em organizações privadas e estatais.

Das 95 observações da pesquisa original, foram descartadas cinco discrepantes ou incompletas, sendo o sujeito respondente ocupante de cargo executivo de primeiro escalão, preferencialmente responsável pelos procedimentos de planejamento e controle estratégico ou gerencial. Os dados sobre a trajetória profissional do sujeito nos levam a crer que não há um viés de percepção associado a faixa etária, educação, experiência pregressa, ramo de atuação etc. Para descrever os fenômenos estudados (Vergara, 1998), adotou-se enfoque quantitativo, baseado na operacionalização de construtos com apoio de análise multivariada.

As questões afins aos indutores de alinhamento estratégico (proposições, fatores e vetores) e ao valor gerado foram testadas a partir da apuração da percepção dos respondentes em escala Likert de seis pontos, buscando evitar-se problema de tendência central. As proposições foram inicialmente submetidas à redução das dimensões latentes, procurando caracterizar construtos-síntese em menor número e capazes de expressar interferências mútuas (Hair et al., 1998). Posteriormente foram computados macroconstrutos pela média algébrica com ponderação entre escores fatoriais e autovalores de cada fator, de modo a definir uma única grandeza adimensional capaz de expressar a variância contida nos blocos de questões. Os testes para diferenças de médias utilizaram métodos não paramétricos, dado que para uma amostra não probabilística e de dimensões reduzidas produzem resultados mais robustos.

\section{Operacionalização}

Ao considerar o intervalo de confiança "indiciário" registrado para aceitação de $\mathrm{H}_{0}, 1$ a partir de 85\%, temos como resultados dos testes $\mathrm{Z}$ de Wilcoxon para diferenças de médias: (i) direcionamento institucional: em sete proposições das 18 foi possível apurar diferenças de 
padrões; (ii) maturidade do planejamento e controle: três proposições confirmam diferenças para 12 testadas; (iii) direcionamento para o futuro: oito proposições em 14 apresentaram diferenças significativas; (iv) direcionamento de recursos humanos: nove proposições testadas apontaram diferenças de padrões entre empresas privadas e estatais; (v) direcionamento da tecnologia da informação: sete proposições entre 11 testadas; (vi) maturidade do gerenciamento da performance: 12 de 25 proposições com diferenças significativas; (vii) performance percebida comparada: 10 em 17.

Ao adotar um ponto de corte mais rigoroso, com intervalo de confiança de 95\%, quatro proposições deixam de apresentar diferenças de médias significativas entre estatais e privadas no tocante ao direcionamento institucional, duas no tocante à maturidade do planejamento e controle, duas no que diz respeito ao direcionamento para o futuro, apenas uma em direcionamento de recursos humanos, quatro em direcionamento da tecnologia da informação, nove na maturidade do gerenciamento da performance e três em performance percebida comparada.

Nas dimensões latentes representativas dos fatores de indução ao alinhamento estratégico (tabela 1), para $\mathrm{p}<0,15$, tem-se que: (i) em direcionamento institucional, dois dos quatro fatores significativos para a diferença de médias; (ii) em maturidade do planejamento e controle, os dois fatores obtidos não significativos; (iii) em direcionamento para o futuro, apenas um; (iv) em direcionamento de recursos humanos, um fator apresenta diferenças de médias significativas; (v) em direcionamento da tecnologia da informação, um fator significativo; (vi) em maturidade o gerenciamento da performance, um fator; e (vii) em performance percebida comparada, apenas diferenciada para as hard capabilities.

Os testes para diferenças de médias nos vetores de alinhamento estratégico (tabela 2), construtos apresentados no apêndice 1, apontam como significativos, para $\mathrm{p}<0,15$, direcionamento institucional, maturidade do planejamento e controle, direcionamento para o futuro, direcionamento de recursos humanos, direcionamento da tecnologia da informação e performance percebida comparada.

$$
\text { Tabela } 1
$$

Teste $\mathrm{W}$ de diferenças de médias para fatores de alinhamento estratégico $(\mathrm{N}=90)$

\begin{tabular}{|lcccc|}
\hline Blocos e Dimensões Latentes & Médias & Médias & $\mathrm{Z}$ & $\mathrm{P}$ \\
$\mathrm{N}=90$ & Privadas & Estatais & & \\
\hline DI Responsabilidade societária & 0,0668 & $-0,3588$ & $-1,801$ & 0,072 \\
DI Ética e prestação de contas & 0,1500 & $-0,4844$ & $-1,516$ & 0,130 \\
DI Responsabilidade ambiental & 0,0577 & $-0,1485$ & $-1,142$ & 0,254 \\
DI Equidade e transparência & $-0,0056$ & 0,0875 & $-0,177$ & 0,859 \\
\hline PC Normatização e mensurabilidade & 0,0751 & $-0,2880$ & $-1,280$ & 0,201 \\
PC Consistência e conciliação & 0,0833 & $-0,3108$ & $-0,945$ & 0,345 \\
\hline
\end{tabular}




\begin{tabular}{|lcccc|}
\hline Blocos e Dimensões Latentes & Médias & Médias & Z & P \\
N = 90 & Privadas & Estatais & & \\
\hline DF Aprendizado e renovação & 0,2079 & $-0,8507$ & $-3,927$ & 0,000 \\
DF Pensamento e coerência & 0,0440 & $-0,1219$ & $-0,157$ & 0,875 \\
DF Proatividade e inovação & $-0,0026$ & $-0,0233$ & $-0,108$ & 0,914 \\
\hline RH Avaliação e retribuição & 0,1983 & $-0,8414$ & $-3,829$ & 0,000 \\
RH Empreendedorismo e aprendizagem & 0,0812 & $-0,2468$ & $-0,925$ & 0,355 \\
TI Conectividade e redesenho & 0,2066 & $-0,8776$ & $-3,691$ & 0,000 \\
TI Administração de dados & 0,0544 & $-0,2525$ & $-1,250$ & 0,211 \\
TI Integração e foco & $-0,0332$ & 0,2517 & $-0,935$ & 0,350 \\
\hline GP Customização & 0,0590 & $-0,2358$ & $-1,535$ & 0,125 \\
GP Premissas & 0,0833 & $-0,3332$ & $-1,211$ & 0,226 \\
\hline PP Hard capabilities & 0,1473 & $-0,5893$ & $-2,845$ & 0,004 \\
PP Soft capabilities & 0,0050 & $-0,0200$ & $-0,030$ & 0,976 \\
\hline
\end{tabular}

Fonte: Elaborado pelos autores.

Tabela 2

Teste $\mathrm{W}$ de diferenças de médias para vetores de alinhamento estratégico $(\mathrm{N}=90)$

\begin{tabular}{|lcccc|}
\hline Blocos e Macroconstrutos & Médias & Médias & & P \\
\hline DI Direcionamento Institucional & Privadas & Estatais & Z & $-1,900$ \\
PC Maturidade Planejamento e Controle & 0,7178 & $-2,3424$ & -057 \\
DF Direcionamento Futuro & 0,5724 & $-2,1679$ & $-1,851$ & 0,064 \\
RH Direcionamento de Recursos Humanos & 0,5647 & $-2,1845$ & $-2,244$ & 0,025 \\
TI Direcionamento da Tecnologia da Informação & 0,9821 & $-3,6935$ & $-3,002$ & 0,003 \\
GP Maturidade do Gerenciamento da Performance & 0,5396 & $-2,0242$ & $-2,116$ & 0,034 \\
PP Performance Comparada & 1,4196 & $-5,6784$ & $-1,063$ & 0,288 \\
\hline
\end{tabular}

Fonte: Elaborado pelos autores.

De uma forma abrangente, foram testados, para as categorias "empresas privadas" e "empresas estatais": (i) 109 "proposições" sobre alinhamento estratégico, com evidências para rejeição de $\mathrm{H}_{0}, 1$ em favor da hipótese alternativa para 56 delas, ou seja, há diferença de médias entre empresas privadas e estatais; (ii) 18 "fatores" de alinhamento estratégico, com evidências para rejeitar $\mathrm{H}_{0}, 1$ em quatro deles; (iii) sete "vetores" de alinhamento estratégico, com evidências para rejeitar $\mathrm{H}_{0}, 1$ em quatro deles. 
Entre todas as proposições pesquisadas, são apresentadas na tabela 3 aquelas que expressam o desempenho das organizações estudadas com base no enfoque de perspectivas múltiplas e balanceadas, comum ao Balanced Scorecard proposto por Kaplan e Norton (1992, 1996, 2001, 2003) e às fontes de originação de valor afins à abordagem de gestão do capital intelectual.

Tabela 3

Teste W para proposições performance percebida comparada $(\mathrm{N}=90)$

\begin{tabular}{|c|c|c|c|c|}
\hline Proposições & $\begin{array}{l}\text { Médias } \\
\text { Privadas }\end{array}$ & $\begin{array}{l}\text { Médias } \\
\text { Estatais }\end{array}$ & Z & $\mathrm{P}$ \\
\hline \multicolumn{5}{|c|}{ Perspectiva Financeira - Capital Financeiro } \\
\hline Giro ativo & 4,44 & 3,39 & $-3,062$ & 0,002 \\
\hline Fluxo de caixa & 4,29 & 3,44 & $-2,434$ & 0,015 \\
\hline Faturamento & 4,60 & 3,72 & $-2,383$ & 0,017 \\
\hline Margem contribuição & 4,25 & 3,44 & $-2,112$ & 0,035 \\
\hline \multicolumn{5}{|c|}{ Perspectiva do Mercado - Capital de Relacionamentos } \\
\hline Valor intangível & 4,38 & 3,67 & $-2,029$ & 0,042 \\
\hline Novos canais & 4,46 & 3,83 & $-1,896$ & 0,058 \\
\hline Reputação & 4,54 & 3,83 & $-2,234$ & 0,026 \\
\hline Novos mercados & 4,31 & 3,94 & $-1,190$ & 0,234 \\
\hline Atendimento demanda & 4,47 & 4,22 & $-0,928$ & 0,353 \\
\hline \multicolumn{5}{|c|}{ Perspectiva dos Processos - Capital Organizacional } \\
\hline Inovação & 4,22 & 3,67 & $-1,612$ & 0,107 \\
\hline Eficácia pontos venda & 3,92 & 3,28 & $-1,632$ & 0,103 \\
\hline Logística & 4,19 & 3,67 & $-1,142$ & 0,253 \\
\hline Faturamento dos fornecedores & 4,04 & 3,61 & $-1,395$ & 0,163 \\
\hline Compliance & 4,42 & 4,50 & $-0,225$ & 0,822 \\
\hline \multicolumn{5}{|c|}{ Perspectiva da Aprendizagem e Crescimento - Capital Humano } \\
\hline Comportamento organizacional & 4,25 & 3,61 & $-2,077$ & 0,038 \\
\hline Inteligência competitiva & 4,18 & 3,78 & $-1,368$ & 0,171 \\
\hline Profissionais estratégicos & 4,29 & 3,11 & $-0,639$ & 0,523 \\
\hline
\end{tabular}

Fonte: Elaborado pelos autores.

Foi perguntado aos sujeitos como havia sido o desempenho comparado entre a empresa observada e seus concorrentes principais nos últimos três exercícios que antecederam à aplicação do survey, em 2002, 2003 e 2004, e as proposições voltavam-se para compor os indicadores de valor - capital financeiro e componentes do capital intelectual (Rezende, 2006).

O desenvolvimento da hipótese 2 , conjecturando sobre a existência de diferentes tipologias de originação de valor entre empresas privadas e estatais, foi empreendido a partir primeiramente da utilização dos agrupamentos hierárquicos identificados por Rezende (2006) para expressar características e padrões diferenciados de alinhamento estratégico.

Foram computados, a partir de rotinas de análise hierárquica de agrupamentos, método de Ward com escores padronizados e utilizada a distância euclidiana ao quadrado, 
três clusters para retratar tipos de empresas no tocante ao gerenciamento dos vetores de alinhamento estratégico.

Os achados quanto aos três agrupamentos foram confirmados por procedimentos uni e multivariados de diferenças de variância (Anova e Manova), pela identificação de pelo menos duas funções de classificação, ambas com $\mathrm{p}<0,01$, e pelo percentual de acertos, superior a 95\% na classificação das observações (Rezende, 2006).

A partir das funções gestão balanceada da performance (91,10\% da variância explicada) e eficiência e ajustamento (explicando 19,90\% da variância) foram posicionadas as observações, resultando em três "tipos" padrão de originação de valor: "empresas ajustadas", "empresas balanceadas" e "empresas desalinhadas".

A tabela 4 apresenta a estratificação das observações conforme origem do capital e padrão de gerenciamento do alinhamento estratégico, registrando a maior concentração das empresas privadas no tipo "ajustada" (59,5\%) e das estatais no tipo "desalinhada" (55,6\%).

Quando os percentuais de estratificação em empresas privadas e estatais são comparados aos percentuais de agrupamento esperados para a amostra como um todo por meio do teste Chi Quadrado, verificam-se diferenças significativas de proporção entre os tipos de organizações no tocante à originação de valor com um nível de significância superior a 99,99\%, levando à rejeição de $\mathrm{H}_{0}, 2$, indicando um padrão diferenciado de acordo com a origem do capital.

Tabela 4

Distribuição das empresas segundo alinhamento estratégico

\begin{tabular}{|c|c|c|c|c|c|c|c|c|}
\hline Grupamentos & \multicolumn{3}{|c|}{ Privadas } & \multicolumn{3}{|c|}{ Estatais } & \multicolumn{2}{|c|}{ Total } \\
\hline Ajustadas & 34 & $37,8 \%$ & $47,2 \%$ & 3 & $3,3 \%$ & $16,7 \%$ & 37 & $41,1 \%$ \\
\hline Balanceadas & 29 & $32,2 \%$ & $40,3 \%$ & 6 & $6,7 \%$ & $33,3 \%$ & 35 & $38,9 \%$ \\
\hline Desalinhadas & 9 & $10,0 \%$ & $12,5 \%$ & 9 & $10,0 \%$ & $50,0 \%$ & 18 & $20,0 \%$ \\
\hline Total & 72 & $80,0 \%$ & $100,0 \%$ & 18 & $20,0 \%$ & $100,0 \%$ & 90 & $100,0 \%$ \\
\hline
\end{tabular}

Fonte: Elaborado pelos autores.

No tocante à hipótese 3 , relativa à existência de "modos" de gerenciamento da originação de valor total a partir dos componentes do capital intelectual e do capital financeiro, foi utilizado o bloco de proposições sobre performance comparada para o estabelecimento de indicadores e índices (Rezende, 2006) capazes de caracterizar dimensões e fluxos fins à criação de valor.

As proposições das dimensões/perspectivas retratadas na tabela 3 foram somadas em cada observação, tendo suas médias calculadas para a criação de indicadores nas dimensões capital de relacionamentos (CR), capital organizacional (CO), capital humano (CH) e Capital financeiro (CF). Foram computados: (i) indicador para capital intelectual (CI) a partir da soma vetorial de 
$\mathrm{CR}, \mathrm{CO}$ e $\mathrm{CH}$; (ii) indicador para valor total a partir da soma vetorial de CI e CF. Finalmente, os indicadores de cada perspectiva foram transformados em distribuições de índices adimensionais por meio da padronização pelo escore Z, com 90 observações comparadas para efeito de diferenças de médias nas categorias empresas privadas e empresas estatais - tabela 5.

Tabela 5

Teste W de diferenças de médias: valor total e componentes

\begin{tabular}{|ccccccrr|}
\hline $\mathbf{N}$ & Índices & $\begin{array}{c}\text { Capital de } \\
\text { Médios }\end{array}$ & $\begin{array}{c}\text { Capital } \\
\text { Organizacional }\end{array}$ & $\begin{array}{c}\text { Capital } \\
\text { Humano }\end{array}$ & $\begin{array}{c}\text { Capital } \\
\text { Intelectual }\end{array}$ & $\begin{array}{c}\text { Capital } \\
\text { Financeiro }\end{array}$ & $\begin{array}{r}\text { Valor } \\
\text { Total }\end{array}$ \\
\hline 72 & Privadas & 0,097 & 0,077 & 0,071 & 0,088 & 0,145 & 0,108 \\
18 & Estatais & $-0,387$ & $-0,308$ & $-0,283$ & $-0,350$ & $-0,581$ & $-0,432$ \\
& Z & $-1,902$ & $-1,562$ & $-1,428$ & $-1,725$ & $-2,688$ & $-2,098$ \\
& $\mathrm{P}$ & 0,057 & 0,118 & 0,153 & 0,085 & 0,007 & 0,036 \\
\hline
\end{tabular}

Fonte: Elaborado pelos autores.

Embora os achados sistematicamente apontem para índices de originação de CI menores nas empresas estatais em todas as dimensões, somente foi possível rejeitar $\mathrm{H}_{0}, 3$ para efeito do capital de relacionamentos na vizinhança de $\mathrm{p}<0,05$, registrada também a rejeição de $\mathrm{H}_{0}, 3$ em favor da existência de diferenças de médias para capital financeiro e valor total.

\section{Discussão}

A figura 2 apresenta uma síntese das proposições, fatores e vetores de alinhamento estratégico em que são enfatizados níveis de significância para diferenças de médias com $\mathrm{p}<0,15$ — intervalo aqui adotado intencionalmente de forma majorada, para fazer frente à proporção entre empresas da amostra e o universo de empresas estatais.

Pela possibilidade de adoção de intervalos de confiança de até 95\% ( $\mathrm{p}<0,05$ ), sobressaem como vetores de alinhamento que mais diferenciam as empresas estatais das empresas privadas: o direcionamento para o futuro, o direcionamento de recursos humanos e o direcionamento da tecnologia da informação.

Como fatores determinantes da distinção entre o menor desempenho das estatais em face das privadas, também para o intervalo de confiança de 95\%, destacam-se: a capacidade de aprender com sua trajetória no ambiente e renovar-se para o futuro; a capacidade de avaliar e retribuir aos colaboradores com base na estratégia formulada; e a capacidade de tornarse mais integrada e conectada pela via do redesenho dos processos do negócio.

A figura 2 apresenta, ainda, proposições representando componentes, construtos e índice de performance geral da estrutura e dinâmica organizacional (Rezende, 2006), registrando nível de desempenho superior, e significante do ponto de vista estatístico, em prol das organizações do setor privado: geração de valor com diferença de média significativa para $\mathrm{p}<0,05$. 


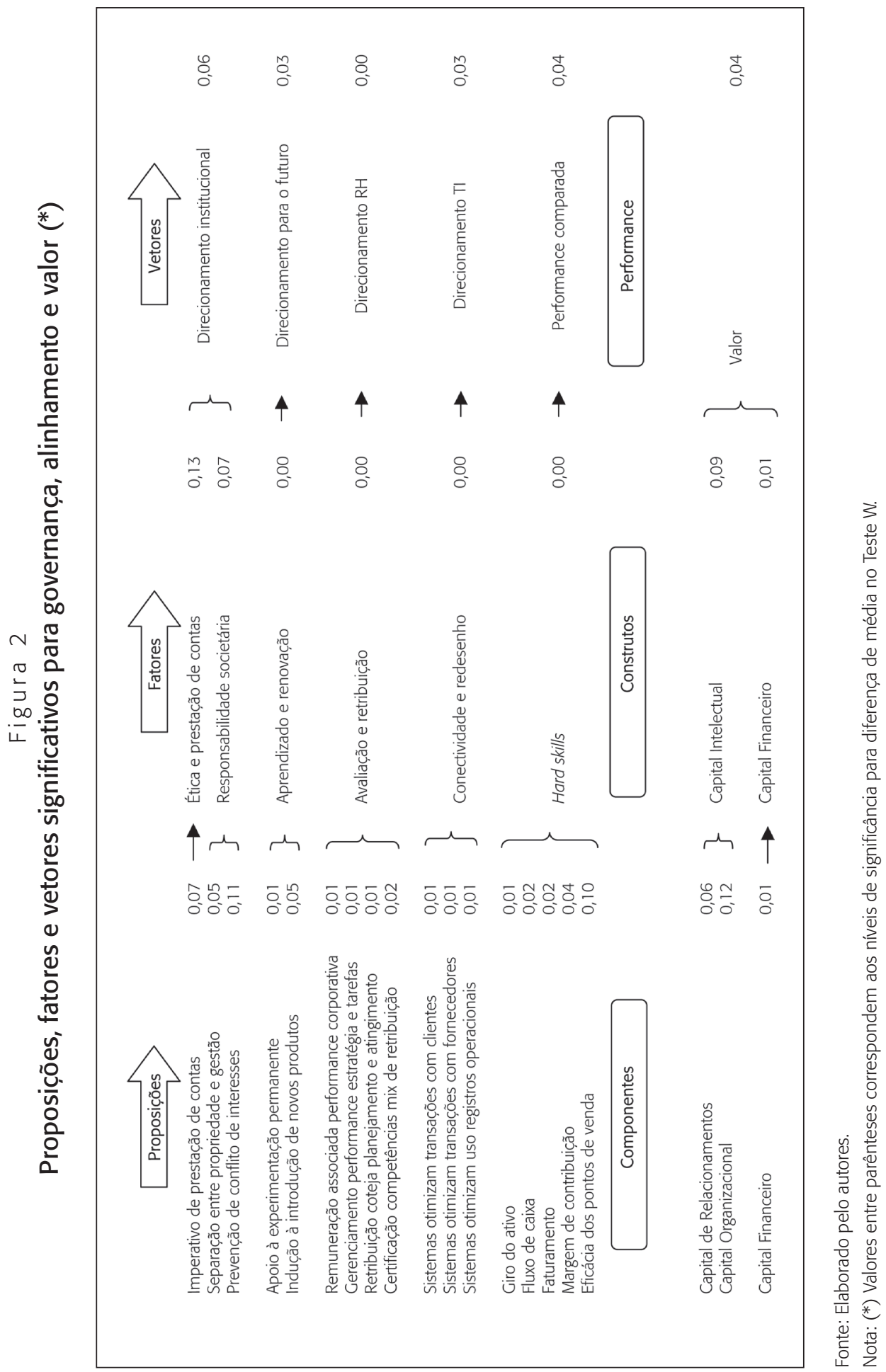

Rev. Adm. Pública - Rio de Janeiro 47(3):695-720, maio/jun. 2013 
O entendimento das diferenças entre a performance das empresas estatais e privadas, tanto pela via do gerenciamento dos vetores de alinhamento estratégico quanto em função dos componentes de valor inerentes à gestão do capital intelectual, pode ser estendido com base nos "tipos" e "modos" identificados durante a operacionalização das hipóteses: empresas "ajustadas", "balanceadas" e "desalinhadas".

As empresas do tipo "ajustadas" - maior participação na amostra e com menor presença de organizações de capital estatal (tabela 4) — dariam ênfase à conformidade e à eficiência dos processos com base na consistência e na conciliação dos objetivos do planejamento e do controle gerencial, na customização de ferramentas para gestão do desempenho e no desempenho das hard capabilities. Usufruem de plataforma de tecnologia da informação estabilizada para as necessidades do negócio, buscando, com algum foco em proatividade e inovação estratégica, alcançar economias de escopo e de escala para suas operações. Ademais, o direcionamento dos recursos humanos estaria mais vinculado a um regime de cumprimento de metas do que ao desenvolvimento profissional — empreendedorismo e aprendizagem — dos trabalhadores, indicando forma de gestão em que a burocracia é utilizada pragmaticamente para promover sintonia e congruência com os objetivos da organização (Ouchi apud Aidemark, 2002:242-243), questão reforçada pela menor atenção para com as Soft Skills e com a coerência do pensamento estratégico.

A pouca ênfase na responsabilidade societária cristalizaria a noção de modelo de controle centralizado e de concentração da propriedade na economia brasileira, o que não deixaria de ser um ponto em comum com a verificação de controle estatal e inibidor de desempenho (Hertog, 2010).

As empresas "ajustadas" seriam viáveis dentro do seu atual ambiente de negócios (Zajac, 2000; Tjan, 2001), mas, diante da pouca atenção para com os vetores de desenvolvimento e da gestão das capabilities para renovar continuamente o valor originado — além do possível enrijecimento pela eficiência em detrimento da flexibilidade - , a garantia da qualidade da performance futura é uma indagação que se faz necessária.

As empresas do tipo "balanceadas" - proporção equivalente de empresas privadas e estatais analisadas na amostra - estariam voltadas para a criação de um futuro exclusivo, baseando sua atuação no desenvolvimento de um time diferenciado de profissionais e na ênfase atribuída às Soft Skills, caracterizando a intenção de criar um habitat próprio, único e compartilhável, típico de empresa viva (De Geus, 1998). Nelas, a tecnologia da informação é um recurso a mais para garantir integração e foco em face das demandas mutantes e embaçadas do novo ambiente de negócios (Davis e Meyer, 1999).

Seriam mais abertas e interessadas na discussão de ideias — premissas e conceitos — do que focadas em coisas - processos e métricas. A complexidade do ambiente seria internalizada pelo rápido reconhecimento dos desvios, das perturbações, do ruído e da instabilidade (Bauer, 1999), sendo o futuro visto como um alvo móvel (Bethlem, 1999) alcançável pela integração de ativos tangíveis, ativos negociáveis, recursos humanos e práticas de gestão (Grouard e Meston, 2001). 
As empresas "balanceadas" refletiriam um segmento empresarial em que o comportamento e as relações são capazes de relativizar o rigor financeiro da mensuração de valor para o shareholder, abrindo espaço para ir além do concreto, do curto prazo e do objetivismo. São empresas balanceadas, no sentido de conseguirem atingir um equilíbrio dinâmico baseado na estratégia (Kaplan e Norton, 2001).

A maior preocupação com o direcionamento institucional deve representar um nível diferenciado de presença do capital na base acionária, mais receptiva e permeável à participação de terceiros. Possivelmente os resultados em relação à responsabilidade societária refletem um estágio de "questão já superada", isto é, as empresas veem na prestação de contas um lugar comum e condição mínima necessária à própria existência.

De uma forma geral, as empresas "balanceadas" possuiriam melhor poder de retratar o modelo de negócios e torná-lo efetivo por meio de decisões táticas (Casadesus-Masanell e Ricart, 2010), principalmente em função de uma maior maturidade na capacidade de explicitar e coordenar expectativas, metas e resultados, evitando a diluição do poder do principal, questão que interessaria sobremaneira à gestão de empreendimentos estatais (Miranda e Amaral, 2011).

As empresas do tipo "desalinhadas" - maior participação percentual relativa das estatais na amostra - encontram-se algo deslocadas em relação às capabilities que originariam valor futuro para organizações, situação possivelmente associada a problemas recentes ou a níveis crescentes de complexidade a que têm sido sujeitadas, caracterizando as empresas em, ou em vias de processo de mudança.

São organizações em oposição às práticas estruturadas de gerenciamento do desempenho e centradas no fazer as atividades determinadas para o presente muito mais do que voltadas para a redefinição da fronteira de negócios - possivelmente por terem um ambiente tarefa definido a priori e sujeito a regulação e normatização abrangentes e rigorosas.

A maior proximidade com a situação de governança financeira do que com a de governança estratégica (Baraldi, 2002) pode ser interpretada por visões alternativas: motivo para a estagnação ou tábua de salvação, visto que "em momentos de crise o mimetismo é um recurso bastante arriscado, pois copiar o que não se sabe como ou por que funciona poderia levar à ampliação do problema" (Bethlem, 1999).

As empresas "desalinhadas" seriam organizações em que a falta de articulação e a configuração de exercício de poder (Mintzberg, 1983) indicariam uma trajetória do estágio de "instrumento" para o de "sistema fechado", em seguida, para o de "arena política" e, finalmente, para o de "renovação". Nesse sentido, seriam prementes intervenções que precipitassem a revisão do modelo de controle e das práticas de governança corporativa (Mintzberg, 1983). Possivelmente, são organizações em que a práxis levou ao isomorfismo sem efetividade e a altos custos de transação, cujos domínios teóricos no campo do novo institucionalismo e da nova economia institucional fogem ao escopo desta pesquisa.

Possivelmente, existem lacunas de competência a serem cobertas (Prahalad e Hamel, 1990; Parry, 1996; Zajac, 2000) que serão decisivas para a revitalização do processo de geração de valor. A aparente necessidade de reconceber fins e meios para fazer frente a uma nova realidade econômica, social, cultural e tecnológica (Tapscott, 1995; Shapiro e Varian, 1998, 
Davis e Meyer, 1999) traz para as empresas com o caráter de "desalinhadas" um desafio premente de busca de alinhamento estratégico aqui e agora como recurso à sobrevivência.

No tocante à "proporção de desalinhamento" das empresas estatais na amostra estudada, poderiam ser levadas em consideração as proposições de Andrés, Guasch e Azumendi (2011) sobre a não aplicação efetiva de conceitos basilares da New Public Management, impedindo a condução do negócio com base em uma estratégia desenhada.

Do ponto de vista do controle das organizações estatais, é notável que estas ainda apresentem procedimentos e padrões de prestação de contas (Louzada, Fontes Filho e Rezende, 2010) aquém dos necessários para um padrão de interação com a sociedade — accountability vertical - , porém, obtemos dos achados deste estudo indícios sobre pontos mais críticos no que tange à melhoria da disclosure.

Sobressai da comparação entre as empresas estatais e privadas, nos três tipos obtidos com base na análise de agrupamentos, um conjunto de desafios de maneira que as estatais se reconfigurem de um modo afim à renovação: é necessário resgatar seu papel instrumental e reforçar seu ambiente tarefa, criando um sistema de indicadores-chave de desempenho capaz de orientar o alcance dos objetivos elementares que justificaram a criação de cada uma delas.

O melhor acompanhamento dos vetores de alinhamento estratégico seria uma resposta razoável ao imperativo de evitar-se o uso das empresas estatais (i) como operadoras de políticas compensatórias: (ii) como simples reguladoras de mercado; e (iii) como organizações "instrumento", desprovidas de uma função de estado.

\section{Considerações finais}

A constatação de que componentes organizacionais e da estratégia interferem na criação de valor, em linha com o senso comum de que as organizações do setor privado seriam mais efetivas, deve ser percebida como ponto de partida, e não de impasse, para a renovação de empresas sob o controle estatal.

Sendo inegável o aumento da importância do valor dos ativos intangíveis em relação ao valor total das organizações, caberia compreender em profundidade a influência que a capacidade de melhor expressar velocidade e intensidade com que as escolhas e a implementação de uma estratégia exercem sobre o fluxo e o estoque de capital intelectual acumulado.

Nesse sentido, a possibilidade de antever, de inferir ou de comparar o valor gerado pela estratégia e pelas operações de uma empresa - a primeira, a partir do alinhamento entre os mecanismos, capacidades e habilidades organizacionais, a segunda, pelo balanceamento entre vetores e indicadores de desempenho - seria oportuna para todos os stakeholders, seja ela estatal ou do setor privado, tendo ainda em conta a possibilidade de expressar com mais proximidade e temporalidade a riqueza existente em uma dada economia ou setor.

Independentemente dos métodos e das metáforas empregadas para materializar o CI, é fácil reconhecer o caráter de dinamicidade envolvido: riqueza oculta que estabelece o arca- 
bouço para o desempenho visível das organizações. Valeria dizer que o padrão de reconhecimento (ou ocultação) poderia sujeitar uma organização, ou tipo de organização, a maiores níveis de entropia, derivada de perda de informação (Tsallis, 2004), e consequentes efeitos no valor originado.

Sobretudo para as empresas estatais, a sistemática de gestão do desempenho por meio de perspectivas múltiplas e integradas - gestão balanceada da performance — favoreceria a preservação do que é central na organização - ideologia e propósitos —, sua evolução - cultura e práticas operacionais - e a construção da visão de futuro - estratégia e metas audaciosas de adição de valor. Além disso, o enfoque equilibrado em torno dos elementos genéricos de organização e gestão que induzem e dão sustentação às vantagens competitivas (proposições, aqui operacionalizadas na forma de fatores e vetores) permitiria alcançar desempenho superior e evitaria que uma empresa caísse na armadilha de dedicar-se unicamente às suas competências distintivas atuais.

Nesse sentido, a criação de mecanismos de transformação estratégica e orquestrada das configurações organizacionais (Mintzberg, Ahlstrand e Lampel, 2000) permitiria o desenvolvimento de capacidades dinâmicas (Hamel, 1996) por meio da interação entre procedimentos, padrões, tipos e modos de interação com os ativos intangíveis e respectivas contribuições na originação de valor.

Com base nas observações estudadas, é possível reforçar a importância dos procedimentos e padrões de direcionamento de recursos humanos como indutores de modos e tipos de organizações que originam valor, principalmente no tocante àqueles referentes à avaliação e retribuição, com registro de diferenças de médias entre empresas privadas e estatais mesmo para $\mathrm{p}<0,01$ (figura 2), também com destaque para os fatores aprendizado e renovação, vinculado ao direcionamento para o futuro, e fator conectividade e redesenho, vinculado ao direcionamento da tecnologia da informação, ambos significativos para $\mathrm{p}<0,03$, consistentes com os achados de Nogueira e Moreira (1998) e de Nogueira (1999) sobre a interação entre a visão do futuro, a gestão de recursos humanos e o emprego da tecnologia da informação no alcance de valor diferenciado.

O que se vislumbra da interpretação das percepções sobre performance alcançada (tabela 5) é a necessidade de as empresas estatais revisarem seus projetos de "criação de valor intangível para os clientes" e de "desenvolvimento de um padrão de comportamento organizacional" que envolva mais e melhor seus colaboradores.

Assim, para além de mandatárias ou missionárias, as organizações estatais poderiam efetivamente atuar na criação das regras do negócio em que atuam desde que com bom poder de interação entre a visão do futuro, o direcionamento dos recursos humanos e a aplicação de tecnologia da informação (Nogueira e Moreira, 1998; Nogueira, 1999).

Nas empresas de capital privado aqui observadas, foi identificado, adicionalmente, um melhor padrão de gerenciamento financeiro em relação às estatais (figura 2), levando à melhor originação final de valor, o que poderia ser creditado a práticas mais maduras ou a melhores níveis, a priori, de capitalização e de disponibilidades (giro do ativo, fluxo de caixa, faturamento e margem de contribuição). 
Os achados relativos às diferenças nos fatores "ética e prestação de contas" e "responsabilidade societária" apresentam desafios adicionais para novos estudos e interpretações sobre direcionamento institucional, visto que mesmo para o "imperativo da prestação de contas" e a "separação entre propriedade e gestão" - que deveriam ser pontos focais da governança das empresas estatais - existem diferenças estatísticas significativas em favor das empresas de capital privado.

Entre todos os fatores gerados pela redução das proposições do estudo de fundo às 18 dimensões latentes, ou seja, as variáveis computadas estatisticamente que explicam a maior parte de como as opiniões dos sujeitos da pesquisa variam, apenas dois mostraram-se superiores para as empresas estatais, porém sequer observam o nível de significância indiciário de p $<0,15$, ou seja, são alertas, mas não evidências: (i) equidade e transparência, no tocante ao direcionamento institucional; e (ii) integração e foco, no tocante ao direcionamento da tecnologia da informação.

Do ponto de vista das contribuições gerais deste estudo, vislumbram-se novas possibilidades de acompanhamento e intervenção no processo de gestão das empresas estatais, pela constatação (i) de recorrentes diferenças de médias nas proposições, fatores e vetores de alinhamento estratégico — refutando-se $\mathrm{H}_{0}, 1$-; (ii) da diferença nas proporções das categorias capital privado e capital estatal para os tipos de originação de valor — refutando-se $\mathrm{H}_{0}, 2$ - ; e (iii) da constatação de modos diferenciados de originação do valor — refutando-se $\mathrm{H}_{0}, 3$ com base no índice de valor total.

Como principais restrições à generalização dos resultados, apontamos (i) a amostragem não probabilística; (ii) o percentual de observações de empresas estatais na amostra; e (iii) a utilização de dados coletados por meio de survey desenvolvido em 2005.

Antevê-se como possibilidades futuras a reaplicação do survey, buscando uma maior adesão das empresas estatais, ou mesmo a aplicação restrita ao universo das estatais para fins de exploração em maior profundidade com o desenvolvimento em paralelo de estudos de casos para outliers.

\section{Referências}

AIDEMARK, Lars-Göan. Balanced scorecard in healthcare; experiences from trials with BSC in five county councils. In: EPSTEIN, Mark; MANZONI, Jean-François (Ed.). Performance measurement and management control: a compendium of research. Oxford: Elsevier Science, 2002. p. 235-272.

ALLEE, Verna. The value evolution: addressing larger implications of an intellectual capital and intangibles perspective. Journal of Intellectual Capital, v. 1, n. 1, p. 17-32, 2000.

ANDRÉS, Luis; GUASCH, Jose L.; AZUMENDI, Sebastian L. Governance in state-owned enterprises revisited: the cases of water and electricity in Latin America and the Caribbean. Policy Research 
Working Paper 5747. The World Bank — Latin American and the Caribbean Region Sustainable Development Unit. August 2011.

BALLOW, John. J.; BURGMAN, Roland; MOLNAR, Michael J. Managing for shareholder value: intangibles, future value and investment decisions. Journal of Business Strategy, v. 25, n. 3, p. 2634, 2004.

BARALDI, Stefano. The balanced scorecard in hospitals: performances measurement as a driver of change - a case study. In: EPSTEIN, Mark; MANZONI, Jean-François (Ed.). Performance measurement and management control: a compendium of research. Oxford: Elsevier Science, 2002. p. 209-234.

BAUER, Ruben. Gestão da mudança: caos e complexidade nas organizações. São Paulo: Atlas, 1999.

BETHLEM, Agrícola S. Gestão de negócios: uma abordagem brasileira. Rio de Janeiro: Campus, 1999.

BOARDMAN, Anthony E.; VINING, Aidan R. Ownership and performance in competitive environments: a comparison of the performance of private, mixed, and state-owned enterprises. Journal of Law and Economics, v. 32, n. 1, p. 1-33, 1989.

BONTIS, Nick; CROSSAN, Mary M.; HULLAND, John. Managing an organizational learning system by aligning stocks and flows. Journal of Management Studies, v. 39, n. 4, p. 438-469, 2002.

BOZEC, Richard; BRETON, Gaétan; CÔTÉ, Louise. The performance of state-owned enterprises revisited. Financial Accountability \& Management, v. 18 n. 4, p. 383-407, 2002.

BRASIL. Decreto-Lei no 200, de 25 de fevereiro de 1967. Dispõe sobre a organização da Administração Federal, estabelece diretrizes para a Reforma Administrativa e dá outras providências. Diário Oficial [da] República Federativa do Brasil, Brasília, DF, 27 fev. 1967. Disponível em: <www.senado. gov.br>. Acesso em: 5 mar. 2006.

BRASIL. Ministério do Planejamento, Orçamento e Gestão. Perfil das empresas estatais federais 2009. Brasília: MP/SE/Dest, 2010a.

BRASIL. Ministério do Planejamento, Orçamento e Gestão. Relatório anual 2009 — execução orçamentária das empresas estatais federais. Brasília: MP/Dest, 2010b.

BUKH, P. N. et al. Multiple integrated performance management systems: IC and BSC in a software company. Singapore Management Review, v. 24, n. 3, p. 21-33, 2002.

CASADESUS-MASANELL, Ramon; RICART, Joan E. From strategy to business models and onto tactics. Long Range Planning, v. 43, p. 195-215, 2010.

CAVALCANTI, Marcos; GOMES, Elisabeth; PEREIRA, Andre. Gestão de empresas na sociedade do conhecimento. Rio de Janeiro: Campus, 2001.

CRAWFORD, Richard. Na era do capital humano: o talento, a inteligência e o conhecimento como forças econômicas; seu impacto nas empresas e nas decisões de investimento. São Paulo: Atlas, 1994. 
DAVIS, Stanley; MEYER, Christopher. Blur: the speed of change in the connected economy. Reading, MA: Addison-Wesley, 1999.

DE GEUS, Arie. A empresa viva. Rio de Janeiro: Campus, 1998.

DEWENTER, Kathryn L.; MALESTA, Paul H. State-owned and privately owned firms: an empirical analysis of profitability, leverage, and labor intensity. The American Economic Review, v. 91, n. 1, p. 320-334, 2001.

DOCA, Geralda. Estatais sem transparência. O Globo, Rio de Janeiro, p. 18, 30 ago. 2010.

EDVINSSON, Leif; MALONE, Michael S. Capital intelectual: descobrindo o valor real de sua empresa pela identificação de seus valores internos. São Paulo: Makron, 1998.

ETHIRAJ, Sendil K.; LEVINTHAL, Daniel. Hoping for A to Z while rewarding only A: complex organizations and multiple goals. Organization Science, v. 20, n. 1, p. 4-21, Jan./Feb. 2008.

FLECK, Denise L. Building up capabilities for sustained corporate growth. In: GLOBALIZATION AND CORPORATE STRATEGIES FOR THE XXI CENTURY: THE BRAZILIAN INNOVATION CHALLENGE, 2005, Rio de Janeiro. Rio de Janeiro: BNDES, 2005. Disponível em: <www.minds.org. br/events/event_2005_3.pdf>. Acesso em: 10 mar. 2008.

GROUARD, Benoit; MESTON, Francis. Empresa em movimento: fundamentos e técnicas da gestão de mudanças. São Paulo: Negócio, 2001.

HAIR JR, Joseph F. et al. Multivariate data analysis. Englewood Cliffs, NJ: Prentice Hall, 1998.

HAMEL, Gary. Strategy as revolution. Harvard Business Review, v. 74, n. 4, p. 69-82, 1996.

HAX, Arnoldo C.; WILDE II, Dean L. The delta model: adaptative management for a changing world. Sloan Management Review, v. 40, n. 2, p. 11-28, 1999.

HERTOG, Steffen. Defying the resource curse: explaining successful state-owned enterprises in rentier states. World Politics, v. 62, n. 2, p. 261-301, 2010.

KAPLAN, Robert S.; NORTON, David P. Organização orientada para a estratégia. Rio de Janeiro: Campus, 2001.

KAPLAN, Robert S.; NORTON, David P. Strategy maps: converting intangible assets into tangible outcomes. Boston: Harvard Business School, 2003.

KAPLAN, Robert S.; NORTON, David P. The balanced scorecard: measures that drive performance. Harvard Business Review, v. 70, n. 1, p. 71-79, 1992.

KAPLAN, Robert S.; NORTON, David P. The balanced scorecard: translating strategy into action. Boston, MA: Harvard Business School, 1996.

KONRAD GROUP. The invisible balance sheet: key indicators for accounting control and valuation of know-how companies. Stockholm: s.n., 2000.

LANGLEY, Ann. Strategies for theorizing from process data. Academy of Management Review, v. 24, n. 4, p. 691-710, 1999. 
LOUZADA, Sergio V.; FONTES FILHO, Joaquim R.; REZENDE, José F. O uso da internet para promoção da accountability pelas empresas estatais federais brasileiras. In: ENCONTRO DE ADMINISTRAÇÃO PÚBLICA E GOVERNANÇA DA ANPAD — Associação Nacional dos Programas de Pós-Graduação em Administração, 4., 2010, Vitória. Anais eletrônicos. Vitória: Anpad, 2010. Disponível em: <www.anpad.org.br/evento.php?acao=trabalho\&cod_edicao_subsecao=641\&cod_evento_edicao $=54 \&$ cod_edicao_trabalho $=12702>$. Acesso em: 10 abr. 2012.

MINTZBERG, Henry. Power in and around organizations. Englewood Cliffs, NJ: Prentice-Hall, INC, 1983.

MINTZBERG, Henry; AHLSTRAND, Bruce; LAMPEL, Joseph. Safári da estratégia. Porto Alegre: Bookman, 2000.

MIRANDA, Rubens A.; AMARAL, Hudson F. Governança corporativa e gestão socialmente responsável em empresas estatais. Revista de Administração Pública, v. 45, n. 4, p. 1069-1094, 2011.

NOGUEIRA, Antonio Roberto R. O alinhamento estratégico, a construção do futuro e a gestão da tecnologia da informação. Tese (doutorado em administração) — Faculdade de Economia e Administração, Universidade de São Paulo, São Paulo, 1999.

NOGUEIRA, Antonio Roberto R.; MOREIRA, Paula C. A. O alinhamento estratégico e a construção do futuro: um estudo exploratório. Revista de Administração Contemporânea [on-line], v. 2, n. 2, p. 103-125, 1998.

NONAKA, Ikujiro; TAKEUCHI, Hirotaka. Criação de conhecimento na empresa: como as empresas japonesas geram a dinâmica da inovação. Rio de Janeiro: Campus, 1997.

NORTON, David P. Benefits based planning. Stage by stage, v. 7, n. 6, 24 p., nov. 1987.

NORTON, David P. Managing benefits from information technology. Information Management, Bradford, v. 3, n. 5, p. 29-35, 1995.

OECD. Organisation for Economic Co-operation and Development. Draft comparative report on corporate governance of state-owned assets. Paris: Daffe/CA/Priv., 2004b. Mimeografado.

OECD. Organisation for Economic Co-operation and Development. Principles of corporate governance. 2004a. Disponível em: <www.oecd.org/dataoecd/32/18/31557724.pdf>. Acesso em: 17 jul. 2005.

PARRY, Scott. The quest for competencies. Training, v. 33, n. 7, p. 48-56, July 1996.

PRAHALAD, C. K.; HAMEL, Gary. The core competence of the corporation. Harvard Business Review, v. 8, n. 3, p. 79-91, may/June 1990.

REZENDE, José F. O alinhamento estratégico, o balanced scorecard e o capital intelectual no Brasil: um estudo empírico nas empresas de maior complexidade e repercussão. Tese (doutorado em ciências da administração) — Instituto Coppead de Administração, Universidade Federal do Rio de Janeiro, Rio de Janeiro, 2006. 
REZENDE, José F.; NOGUEIRA, Antonio Roberto R. Strategic alignment, performance, and value. Latin American Business Review, v. 11, n. 4, p. 339-363, 2010.

ROOS, Johan et al. Intellectual capital: navigating the new business landscape. Londres: MacMillan Press, 1997.

SERENKO, Alexander; BONTIS, Nick. Meta-review of knowledge management and intellectual capital literature: citation impact and research productivity rankings. Knowledge and process management, v. 11, n. 3, p. 185-198, 2004.

SHAPIRO, Carl; VARIAN, Hal R. Information rules: a strategic guide to the network economy. Boston, MA: Harvard Business School Press, 1998.

STEWART, Thomas A. Capital intelectual: a nova vantagem competitiva das empresas. Rio de Janeiro: Campus, 1998.

SVEIBY, Karl-Erik. Measuring intangibles and intellectual capital: an emerging first standard. Disponível em: <www.sveiby.com.au/library>. Acesso em: 14 ago. 1998.

SVEIBY, Karl-Erik. Towards a knowledge perspective on organization. Tese (doutorado em business administration) - Department of Business Administration, University of Stockholm, Estocolmo, 1994.

TAPSCOTT, Don. The digital economy: promise and peril in the age of networked intelligence. Nova York: McGraw-Hill, 1995.

TJAN, Anthony K. Finally a way to put your internet portfolio in order. Harvard Business Review, v. 79, n. 2, p. 76-85, Feb. 2001.

TSALLIS, Constantino. Entrevista: uma forma de explicar a bolsa e a geladeira. O Globo, Rio de Janeiro, 2 fev. 2004. Caderno Ciência e Vida, p. 22.

VERGARA, Sylvia C. Projetos e relatórios de pesquisa em administração. São Paulo: Atlas, 1998.

ZAJAC, Edward J.; KRAATZ, Matthew S.; BRESSER, Rudi K. F. Modeling the dynamics of strategic fit: a normative approach to strategic change. Strategic Management Journal, Chichester, v. 21, n. 4, p. 429, Apr. 2000.

José Francisco de Carvalho Rezende é doutor em ciências da administração pelo Coppead, Universidade Federal do Rio de Janeiro (UFRJ), e professor adjunto do Programa de Pós-Graduação da Universidade do Grande Rio (PPGA/Unigranrio). E-mail: rezende.jose@unigranrio.br.

Joaquim Rubens Fontes Filho é doutor em administração e professor adjunto da Escola Brasileira de Administração Pública e de Empresas da Fundação Getulio Vargas (EBAPE/FGV). E-mail: joaquim. rubens@fgv.br. 


\section{Apêndice 1}

Testes de validação dos vetores de alinhamento estratégico

\begin{tabular}{|lcccccc|}
\hline Macroconstruto N = 90 & Itens & Fatores & KMO & Bartlett & Alpha & Variância \\
\hline DI Direcionamento Institucional & 18 & 4 & 0,829 & 0,000 & 0,866 & 63,68 \\
PC Maturidade Planejamento e Controle & 12 & 2 & 0,824 & 0,000 & 0,906 & 66,21 \\
DF Direcionamento para o Futuro & 14 & 3 & 0,859 & 0,000 & 0,841 & 63,76 \\
RH Direcionamento RH & 12 & 2 & 0,911 & 0,000 & 0,903 & 65,09 \\
TI Direcionamento TI & 11 & 3 & 0,840 & 0,000 & 0,905 & 78,89 \\
GP Maturidade Gestão da Performance & 25 & 2 & 0,952 & 0,000 & 0,983 & 78,41 \\
PP Performance Comparada & 17 & 2 & 0,944 & 0,000 & 0,965 & 73,09 \\
Totais & 109 & 18 & & & & \\
\hline
\end{tabular}

Fonte: Elaborado pelos autores.

\section{Apêndice 2}

Resumo dos testes W de diferenças de médias

\begin{tabular}{|c|c|c|c|c|c|c|c|c|c|c|c|c|}
\hline \multirow{3}{*}{$\begin{array}{l}\text { Blocos Pesquisados } \\
\text { Intervalos de confiança }\end{array}$} & \multicolumn{4}{|c|}{ Proposições } & \multicolumn{4}{|c|}{ Fatores } & \multicolumn{4}{|c|}{ Vetores } \\
\hline & \multirow{2}{*}{ \# } & \multicolumn{3}{|c|}{ rejeitadas $\mathrm{H}_{0}$} & \multirow{2}{*}{ \# } & \multicolumn{3}{|c|}{ rejeitados $\mathrm{H}_{0}$} & \multirow{2}{*}{ \# } & \multicolumn{3}{|c|}{ rejeitados $\mathrm{H}_{0}$} \\
\hline & & $85 \%$ & $90 \%$ & $95 \%$ & & $85 \%$ & $90 \%$ & $95 \%$ & & $85 \%$ & $90 \%$ & $95 \%$ \\
\hline Direcionamento Institucional & 18 & 7 & 6 & 3 & 4 & 2 & 1 & 0 & 1 & 1 & 1 & 0 \\
\hline Planejamento e Controle & 12 & 3 & 1 & 1 & 2 & 0 & 0 & 0 & 1 & 1 & 1 & 0 \\
\hline Direcionamento para o Futuro & 14 & 8 & 7 & 6 & 3 & 1 & 1 & 1 & 1 & 1 & 1 & 1 \\
\hline Recursos Humanos & 12 & 9 & 8 & 8 & 2 & 1 & 1 & 1 & 1 & 1 & 1 & 1 \\
\hline Tecnologia da Informação & 11 & 7 & 5 & 3 & 3 & 1 & 1 & 1 & 1 & 1 & 1 & 1 \\
\hline Gerenciamento da Performance & 25 & 12 & 7 & 3 & 2 & 1 & 0 & 0 & 1 & 1 & 0 & 0 \\
\hline Performance Comparada & 17 & 10 & 8 & 7 & 2 & 1 & 1 & 1 & 1 & 1 & 1 & 1 \\
\hline
\end{tabular}

Fonte: Elaborado pelos autores.

Nota: \# = quantidade de itens testados. 